\title{
SPECTRAL ANALYSIS OF EXCHANGE RATES
}

\author{
ALEŠA LOTRIČ DOLINAR ${ }^{1}$ \\ Faculty of Economics, University of Ljubljana, Kardeljeva ploščad 17, SI-1000 Ljubljana, SLOVENIA
}

\begin{abstract}
Using spectral analysis is very common in technical areas but rather unusual in economics and finance, where ARIMA and GARCH modeling are much more in use. To show that spectral analysis can be useful in determining hidden periodic components for high-frequency finance data as well, we use the example of foreign exchange rates.
\end{abstract}

Key-Words: - Spectral Analysis, Frequency Domain, Finance, Exchange Rates

\section{Introduction}

Modern time series analysis consists of an enormous number of different analytic methods (such as trends, ARIMA models, GARCH models, spectral analysis, neural networks, and many others), most of which are basically divided into two large families. One approach, which is more commonly used, is the so-called "correlation" or "time domain" analysis, whereas the other is the "spectral" or "frequency domain" approach. Although the two ways are very different, they are closely linked.

Usually, time series analysis in economics or business area (as the one presented) considers stochastic, or random, processes. Therefore, not only the values of the process are of our interest, but also its variance.

Spectral analysis offers an additional insight into the time series behavior and structure of its variance. It is also essential in identifying hidden periodic fluctuations.

\footnotetext{
1. alesa.1.dolinar@ef.uni-lj.si http://www.ef.uni-lj.si/person/Alesa-Lotric-Dolinar
} 


\section{Spectral Analysis}

The name for spectral analysis comes from a very simple spectral analysis performed very frequently in our every-day life, namely, defining a colour of a certain object, where the usual definition of a color is wavelength or frequency of light. Besides, the colors are usually not pure colors, but their combinations. While deciding about the color of a certain object and about which color prevails, we perform spectral analysis. For more precise decomposition of light, we send it through a prism, resulting in a spectrum of colors.

Similar analysis can also be performed for phenomena from other areas. The process under our investigation only has to be stationary, i. e. it has to fluctuate around certain value and be stable up to a certain degree (no obvious positive or negative trend). Very commonly, spectral analysis is employed in the field of physics (optics, acoustics, fluid mechanics, turbulence), electrical engineering, geophysics (oceanography, seismology), medicine (EEG and ECG),... As micro-level economic and especially financial data are observed at ever greater frequency (on an almost continuous basis), the spectral analysis can be applicable here as well.

Unfortunately, the literature on spectral analysis in economy and finance is rather scarce, as opposed to ARIMA or ARCH methods, for example. Another problem is that the terminology is quite different with different authors, let alone the notations. Next, some authors operate with frequency $f$ (where the length of the entire period is 1 ), while the others with angular frequency $\omega=2 \pi f$ (with $2 \pi$ as the length of the period).

Anyway, spectral or frequency-domain analysis is basically trying to determine how important cycles of different frequencies are for the behavior of a certain time series. Essentially it has to do with decomposition of a stationary process into a sum of sinusoidal components with uncorrelated random coefficients. We consider the observed time series as (according to Fourier theorem) a weighted sum of underlying series that have different cyclical patterns, i.e. as a Fourier series, which is a linear combination of sinusoids of different frequencies and amplitudes. Thus, the total variance of the observed time series can be viewed as a sum of the contributions of these underlying series, which vary at different frequencies. Therefore, spectral analysis is usually applied in order to decompose the observed time series variance.

A device that uses this idea is periodogram, which was introduced already at the end of $19^{\text {th }}$ century by A. Schuster [2], originally to detect periodicities, hidden in noise.

\subsection{Spectrum and Periodogram}

Let $y_{t}, t=-\infty, \ldots, \infty$ be the observed zero mean, stationary time series process. The autocovariance at lag $\mathrm{k}$ is defined as $\lambda_{k}=\lambda_{-k}=\operatorname{Cov}\left(y_{t}, y_{t-k}\right)$. The autocovariance-generating function for this time-series process is $g_{Y}(z)=\sum_{k=-\infty}^{\infty} \lambda_{k} z^{k}$. If we evaluate this function at the complex value $z=e^{-i \omega}, \omega$ real, and divide it by $2 \pi$ we obtain the (population) spectrum of the observed time-series process.

According to Wiener-Khintchine theorem [2], for any stationary stochastic process with auto covariance function $\lambda_{k}$, there exists a monotonically increasing function $F(\omega)$, such that

$\lambda_{k}=\int_{0}^{\pi} \cos (\omega k) d F(\omega)$. This is the so-called spectral representation of the autocovariance function, where $F(\omega)$ can be interpreted as the contribution to the variance of the original series, accounted for by frequencies in the range $(0, \omega)$. Spectral density function, or spectrum, is then defined as 


$$
f(\omega)=\frac{d F(\omega)}{d \omega}
$$

where the spectrum represents the contribution to variance of the components with frequencies in the range $(\omega, \omega+d \omega)$. It shows relative importance of a certain frequency. The total area under the spectrum curve equals the total variance of the original process.

The spectrum is Fourier transform of $\lambda_{k}$ and $\lambda_{k}$ is (inverse) Fourier transform of $f(\omega)$. The Fourier transform pair is thus:

$$
\begin{aligned}
& f_{Y}(\omega)=\frac{1}{2 \pi} \sum_{-\infty}^{\infty} \lambda_{k} e^{-i \omega k} \\
& \lambda_{k}=\int_{-\pi}^{\pi} e^{i \omega k} f_{Y}(\omega) d \omega
\end{aligned}
$$

The spectrum can be simplified (due to symmetry of autocovariances and sine and cosine functions, DeMoivre's theorem and known polar values [3]) to

$$
f_{Y}(\omega)=\frac{1}{2 \pi}\left(\lambda_{0}+2 \sum_{k=1}^{\infty} \lambda_{k} \cos (\omega k)\right),
$$

which is a strictly real continuous function of $\omega$. The cosine function is cyclic with period $2 \pi$, which means that the entire spectrum is known if the values for $\omega$ from 0 to $\pi$ are known. (Due to the symmetry of cosine function, it suffices to know the values of $\omega$ from 0 to $\pi$, not to $2 \pi$ - the values of the spectrum for $\omega$ from 0 to $-\pi$ are thus the same as those from 0 to $\pi$.) If the data-generating process actually includes cycles with frequencies greater than $\pi$, these will be imputed to cycles with frequencies between 0 and $\pi$, which is called aliasing [4]. The highest frequency about which we can get some meaningful information from the dataset, is called Nyquist frequency ( $\pi$, or 0,5 if not angular). The lower the frequency we are interested in, the longer the time horizon over which we need to take measurements (so as to distinguish between a trend and periodic movement), whereas the higher the frequency we are interested in, the more frequently we have to take observations.

From

$$
\lambda_{k}=\int_{-\pi}^{\pi} f_{Y}(\omega) \cos (k \omega) d \omega=2 \int_{0}^{\pi} f_{Y}(\omega) \cos (k \omega) d \omega
$$

we can see that the spectral density function and the sequence of autocovariances only produce two different aspects of the same time-series process. The spectrum and the sequence of autocovariances contain exactly the same information.

The spectrum is related to the whole population, or the whole realization. Usually we are only presented with a part of it, which can be considered a sample. The sample counterpart to the spectrum is a periodogram

$$
\hat{f}_{Y}(\omega)=\frac{1}{2 \pi}\left(c_{0}+2 \sum_{k=1}^{T-1} c_{k} \cos (\omega k)\right)
$$

which is a discrete Fourier transform of the complete sample autocovariance function

$$
\begin{aligned}
& c_{k}=c_{-k}=\frac{1}{T} \sum_{t=k+1}^{T}\left(y_{t}-\bar{y}\right)\left(y_{t-k}-\bar{y}\right) \\
& k=0,1, \ldots, T-1 \\
& \bar{y}=\frac{1}{T} \sum_{t=1}^{T} y_{t}
\end{aligned}
$$


But a periodogram is not a consistent estimator of $f(\omega)$, as its variance does not decrease with increasing number of observations (we need $T$ observations to evaluate $T$ parameters in Fourier representation). One solution is smoothing of the periodogram and thus removing irregular variation. Sometimes smoothing is called kernel estimation, window estimation, or lag windowing (a set of smoothing weights is called a window). The revised estimator is thus

$$
\hat{f}_{Y}(\omega)=\frac{1}{2 \pi}\left(w_{0} c_{0}+2 \sum_{k=1}^{M} w_{k} c_{k} \cos (\omega k)\right),
$$

where $M=2 \sqrt{T}$ roughly if also truncation is performed [2]. There are several suggestions for weights [2]: Bartlett, Hamming, Hann, Parzen, Tukey,... We use Hamming (or Tukey-Hamming):

$$
\hat{f}_{Y}(\omega)=0,23 \hat{f}_{1}(\omega-\pi / M)+0,54 \hat{f}_{1}(\omega)+0,23 \hat{f}_{1}(\omega+\pi / M)
$$

where $\hat{f}_{1}(\omega)$ equals to the equation (1) with all weights $w_{k}$ set to 1 .

When testing for periodicity at the confidence level of $\alpha$, we get the confidence interval of $(r * \hat{f}(\omega) / b, r * \hat{f}(\omega) / a)$, where $a=\chi^{2}{ }_{\alpha}(r), \quad b=\chi^{2}{ }_{1-\alpha}(r)$ and $r=2.516 \mathrm{~N} / M$ for the Hamming window [6].

\section{Application of Spectral Analyisis to GBP/EUR and TRY/EUR Exchange Rates}

For comparison, we apply spectral analysis to two Euro foreign exchange rate time series, British Pound (GBP/EUR) and Turkish Lira (TRY/EUR). The data for British Pound exchange rate were available from the beginning of 1999 on, which resulted in 3716 daily observations (up to July $4^{\text {th }}$, 2013), whereas the data for Turkish Lira exchange rate were available from the beginning of 2005 on, making 2179 daily observations (Fig. 1).

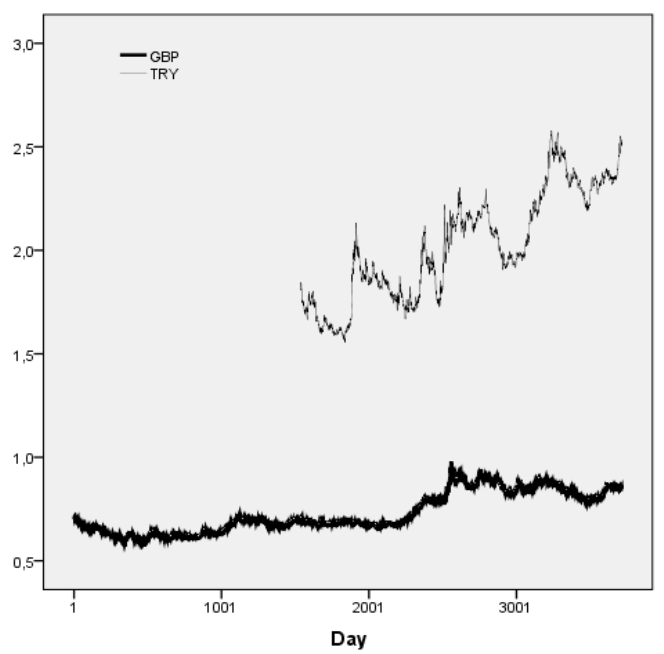

Figure 1. GBP/EUR and TRY/EUR exchange rates from 1999 and 2005 on, respectively

In order to make both time series stationary we took first differences and conducted the analysis on the transformed time series.

Fig. 2 represents the periodogram for GBP/EUR exchange rate, Fig. 3 the smoothed periodogram for GBP/EUR exchange rate, plotted against frequency, and Fig. 4 the smoothed periodogram for GBP/EUR exchange rate, plotted against period. When considering the two smoothed periodograms simultaneously, the interpretation is easier. Likewise, Figs. 5, 6 and 7 represent the same for TRY/EUR exchange rate. In Figs. 3 and 6, frequency ranges from 0 (for a constant mean) to 0,5 (for a cycle with only 2 observations). In Figs. 4 and 7, period ranges from 2 (for a cycle with only 2 
observations) to the number of all observations (for a constant mean). Period is displayed in a power scale.

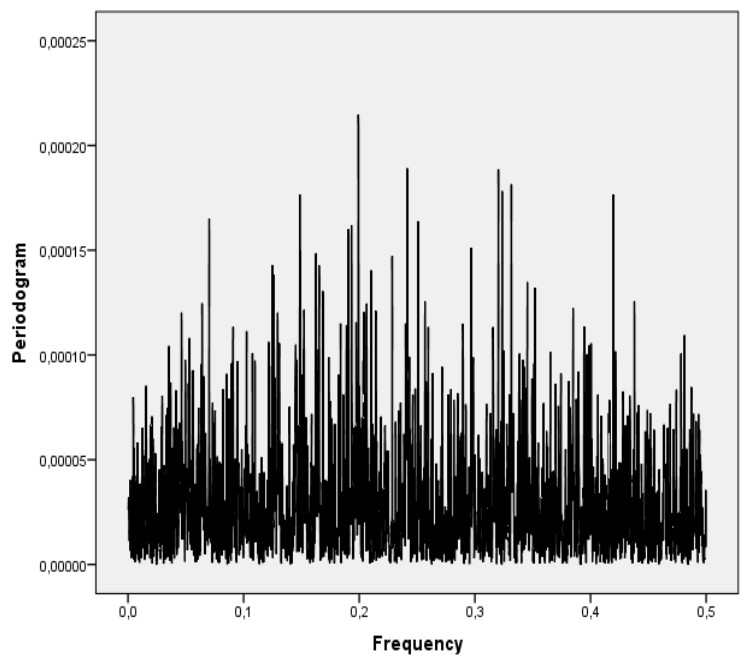

Figure 2. Periodogram for first differences of GBP/EUR exchange rate

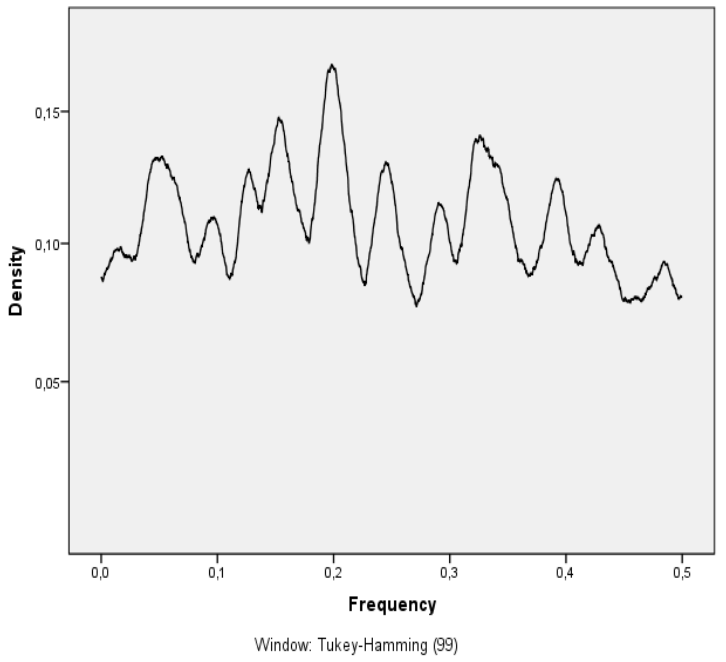

Figure 3. Smoothed periodogram for first differences of GBP/EUR exchange rate, plotted against frequency 


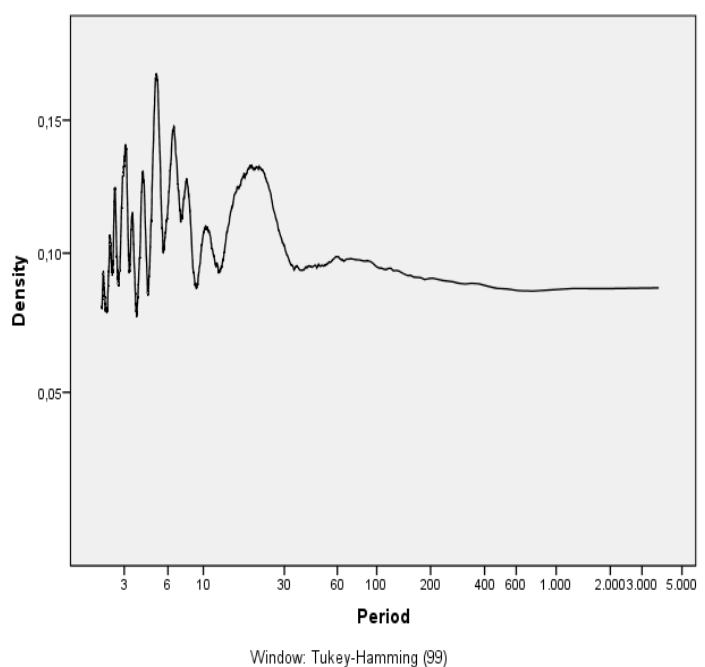

Figure 4. Smoothed periodogram for first differences of GBP/EUR exchange rate, plotted against period

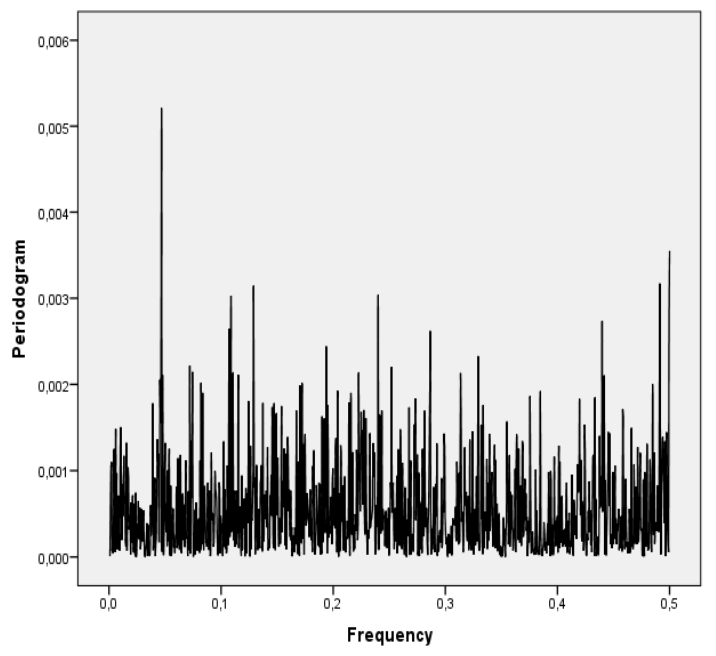

Figure 5. Periodogram for first differences of TRY/EUR exchange rate

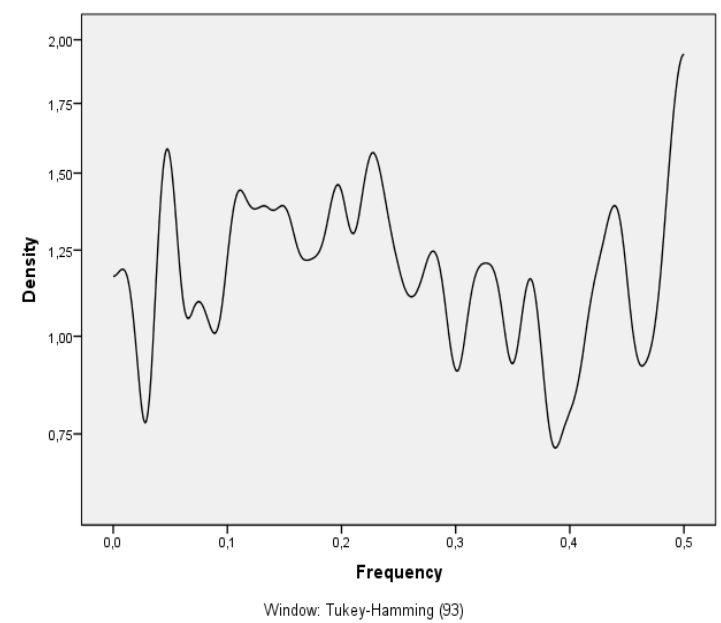

Figure 6. Smoothed periodogram for first differences of TRY/EUR exchange rate, plotted against frequency 


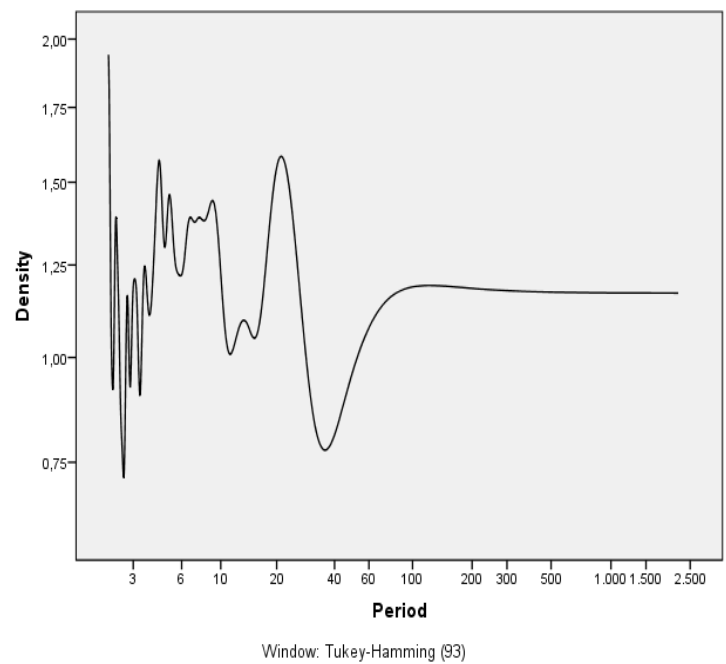

Figure 7. Smoothed periodogram for first differences of TRY/EUR exchange rate, plotted against period

Sine- or cosine- shaped periodic components appear in the periodogram as single peaks, whereas nonsinusoidal periodic components appear as a series of equally spaced peaks of different amplitudes as can be seen from Fig. 3 for GBP/EUR exchange rate. We also notice that (see Figs. 3 and 4) the highest peak, which represents the greatest share of variance, is around frequency 0,2 corresponding to the period length of $5(=1 / 0,2)$ (working) days, indicating weekly periodicity.

For TRY/EUR exchange rate we observe (see Figs. 6 and 7) the highest peak at the highest frequency, suggesting that the major part of variance is represented by day-to-day volatility. Otherwise, the highest peak for a "true" periodicity is around frequency 0,05 corresponding to the period length of 1 month $(1 / 0,05=20$ working days $)$, revealing the prevailing monthly fluctuations.

\section{Conclusion}

The autocorrelation function (which is a base of the ARIMA and GARCH models) and the spectrum are transforms of each other, and therefore they are mathematically equivalent in ways of describing a stationary stochastic process. Thus, the only question is their representational value.

From a practical point of view, they are complementary to each other, rather than excluding one another. When being interested in the main building blocks of the variance and searching for some meaningful interpretation, the spectral analysis is irreplaceable. Or, if we try to detect multiple underlying periodicities and/or a lot of noise, spectral analysis shows the clearest picture. Besides, it can also be used as informal testing for white noise that should have a constant spectrum.

On the other hand, when trying to predict the actual values, the time-domain analysis may prove to be, above all, less complicated, especially due to somewhat less intuitional concept of the frequency-domain analysis, and even more so due to the fact that we are usually not taught the spectral analysis during the basic statistics or econometrics courses.

\section{References}

Anderson T. W., The Statistical Analysis of Time Series, John Wiley \& Sons, 1994

Chatfield C., The Analysis of Time Series, Chapman \& Hall/CRC, 1999

Greene W. H., Econometric Analysis, Prentice Hall, 2000

Hamilton J. D., Time Series Analysis, Princeton University Press, 1994

IBM SPSS Statistics - Online Help

(http://127.0.0.1:1319/help/index.jsp?topic=/com.ibm.spss.statistics.help/overvw_auto_0.htm) 
Lotrič Dolinar A.: The Slovenian Stock Market Index (SBI20 - Slovenski borzni indeks) from the Aspect of Frequency Domain, in Forecasting Financial Markets: Thjeory and Applications (ed. Milo W., Wdowiński P.), Wydawnictwo Uniwersytetu Łódzkiego, 2005

Madsen H., Time Series Analysis, Chapman \& Hall/CRC, 2008

Source:

http://www.ecb.int/stats/exchange/eurofxref/html/index.en.html, 4.7.2013 